\title{
Improvement in Peripheral Glucose Uptake After Gastric Bypass Surgery Is Observed Only After Substantial Weight Loss Has Occurred and Correlates with the Magnitude of Weight Lost
}

\author{
Guilherme M. Campos • Charlotte Rabl • Sofia Peeva • \\ Ruxandra Ciovica • Madhu Rao • Jean-Marc Schwarz • \\ Peter Havel • Morris Schambelan • Kathleen Mulligan
}

Received: 29 May 2009 / Accepted: 29 September 2009 / Published online: 17 October 2009

(C) 2009 The Author(s). This article is published with open access at Springerlink.com

\begin{abstract}
Introduction Altered gut and pancreatic hormone secretion may bolster resolution of insulin resistance after Roux-en-Y gastric bypass (RYGB), but the independent effects of weight loss and hormonal secretion on peripheral glucose disposal are unknown. Methods Two groups of nondiabetic morbidly obese patients were studied: RYGB followed by standardized caloric restriction (RYGB, $n=12$ ) or caloric restriction alone (diet, $n=10$ ). Metabolic evaluations (euglycemic-hyperinsulinemic clamp, meal tolerance test) were done at baseline and 14 days (both groups) and 6 months after RYGB.

Results At baseline, body composition, fasting insulin, and glucose and peripheral glucose disposal did not differ between groups. At 14 days, excess weight loss (EWL) was similar (RYGB, 12.7\% vs. diet, 10.9\%; $p=0.12$ ), fasting insulin and glucose decreased to a similar extent, and RYGB subjects had altered postmeal patterns of gut and pancreatic hormone secretion. However, peripheral glucose uptake ( $M$ value) was unchanged in both groups. Six months after RYGB, EWL was $49.7 \%$. The changes in fasting glucose and insulin levels and gut hormone secretion persisted. $M$ values improved significantly, and changes in $M$ values correlated with the $\% \operatorname{EWL}(r=0.68, p=0.02)$.

Conclusions Improvement in peripheral glucose uptake following RYGB was observed only after substantial weight loss had occurred and correlated with the magnitude of weight lost.
\end{abstract}

This paper was presented at the 50th Annual Meeting of the Society for Surgery of Alimentary Tract during Digestive Disease Week, Chicago, IL, June 2009.

G. M. Campos $\cdot$ C. Rabl $\cdot$ S. Peeva $\cdot$ R. Ciovica

Department of Surgery, University of California San Francisco,

San Francisco, CA, USA

M. Rao $\cdot$ J.-M. Schwarz $\cdot$ M. Schambelan $\cdot$ K. Mulligan

Department of Medicine, University of California San Francisco,

San Francisco, CA, USA

P. Havel

Department of Nutrition, University of California Davis,

Davis, CA, USA

G. M. Campos $(\bowtie)$

Department of Surgery, School of Medicine and Public Health, University of Wisconsin,

600 Highland Avenue, H4/744 CSC,

Madison, WI 53792-7375, USA

e-mail: campos@surgery.wisc.edu
Keywords Bariatric surgery - Insulin resistance - Obesity · Morbid obesity · Gastric bypass · Weight loss · GLP-1 . Insulin · Type 2 diabetes · Diabetes · Calorie restriction . Incretin

\section{Introduction}

Several bariatric surgical techniques originally designed to promote weight loss offer a variable but impressive rate of cure for type 2 diabetes mellitus (T2DM). In $80 \%$ of patients who undergo Roux-en-Y gastric bypass surgery (RYGB), T2DM resolves or improves significantly. ${ }^{1,2}$ RYGB is the most common bariatric surgical technique used in the USA and seems to provide better weight loss and higher rates of resolution of T2DM than purely restrictive techniques such as laparoscopic gastric banding. ${ }^{3-5}$ 
The proposed mechanisms to account for why RYGB offers this remarkable rate of resolution of T2DM have been under extensive scrutiny in both animals and humans. ${ }^{1,6-12}$ At the center of the debate is the relative contribution of greater and sustained weight loss, or to an altered pattern of gut and pancreatic hormone secretion, frequently called the "incretin effect", or other factors. ${ }^{13,14}$ RYGB creates an anatomical rearrangement that delivers a partially digested food bolus directly into the second portion of the small bowel while avoiding contact with a large portion of the stomach and the duodenum. ${ }^{15-17}$ This, in turn, results in altered glucose kinetics ${ }^{18}$ and altered secretion of many gut and pancreatic hormones known to affect glucose metabolism ${ }^{6,19}$ and has additional effects on gastric emptying ${ }^{20}$ and on neurohormonal gut-brain signaling that regulates energy homeostasis and hungersatiety mechanisms. ${ }^{10,20,21}$ However, to date, there have been few detailed and controlled metabolic studies of the interplay and independent effects of RYGB on the many factors that affect insulin resistance and glucose metabolism, such as beta cell function, the associated changes on gut and pancreatic hormone levels, the magnitude and rate of weight loss, energy balance, changes in body composition, and other factors. Information from such studies might help clinicians and patients in choosing among available surgical treatments for morbid obesity and guide the search for novel surgical procedures to treat obesityassociated T2DM in patients with lower body mass indices (BMI). Therefore, the goal of this study was to delineate short-term changes in total body glucose disposal, gut and pancreatic hormone secretion, and body composition, while controlling for energy balance and delineate the same changes after more substantial weight loss had occurred 6 months after RYGB.

\section{Patients and Methods}

Morbidly obese patients, selected to undergo gastric bypass surgery (RYGB), were recruited at the University of California, San Francisco's (UCSF) Bariatric Surgery Program. They met the National Institute of Health and UCSF Bariatric Surgery Program criteria for bariatric surgery: age 21 to 65 years old, BMI either $>40$ or $>35 \mathrm{~kg} / \mathrm{m}^{2}$ with high-risk comorbidities, documented desire to undergo bariatric surgery, well informed and motivated, acceptable operative risks, evaluated and cleared for the procedure by a certified dietitian and a psychiatrist or psychologist, documented repeated failure of nonsurgical supervised weight loss programs, documented BMI $>35 \mathrm{~kg} / \mathrm{m}^{2}$ for more than 5 years, and ability and willingness to provide informed consent. Exclusion criteria included previous weight loss surgery; a previous esophageal, gastric, pancreatic, adrenal, small bowel, large bowel, thyroid, or central nervous system operation; diagnosis of thyroid, liver, pancreatic, adrenal, hypothalamic, pituitary, ovarian, and chronic renal disease; diagnosis of T2DM; use of insulin or any oral medications for T2DM; or unwillingness or inability to give informed consent. This project was approved by the UCSF Committee of Human Research and the UCSF Clinical and Translational Science Institute Clinical Research Center (CRC) Advisory Committee.

\section{Randomization and Metabolic Evaluation}

Twenty-two patients were allocated to two groups: One underwent immediate laparoscopic RYGB surgery followed by standardized calorie restriction (RYGB, $N=12$ ), and the other underwent caloric restriction only (diet, $N=10$ ). Allocation to either group was determined by randomization in the initial 17 patients studied and then by CRC and surgery date availability in the last five patients.

All participants underwent the same baseline metabolic evaluation (visit 1, V1). Subjects were admitted to the CRC at 7:00 p.m. on study day 0 for an initial complete medical history and physical examination. On that day, they began an "ad libitum" diet up to a maximum intake of $25 \mathrm{kcal} / \mathrm{kg} /$ $24 \mathrm{~h}$. Each morning upon arising, they were weighed on a calibrated scale after voiding.

Meal Tolerance Test On day 1, participants underwent a meal tolerance test (MTT), which consisted of a standardized $300 \mathrm{kcal}$ in $100 \mathrm{~mL}$ liquid meal containing $50 \%$ carbohydrate, $30 \%$ protein, and $20 \%$ fat with $9.9 \mathrm{~g}$ of simple sugars in a total of $38 \mathrm{~g}$ carbohydrate, $10 \mathrm{~g}$ of fat, and $15 \mathrm{~g}$ of protein. Participants were asked to consume this meal within a maximum of $20 \mathrm{~min}$. Blood samples, obtained through an intravenous catheter inserted in the forearm, were drawn at $-120,-60,-5,0,+5,+15,+30,+60+120$, and $+180 \mathrm{~min}$ relative to the start of the meal. After collection, the samples were processed on site and stored at $-70^{\circ} \mathrm{C}$ for subsequent batch analysis of glucose, insulin, glucagon-like peptide-1 (GLP-1), and glucose-dependent insulinotropic polypeptide (GIP; formerly known as gastric inhibitory polypeptide).

Euglycemic-Hyperinsulinemic Clamp On day 2, after an overnight fast, peripheral glucose uptake was measured by the hyperinsulinemic-euglycemic clamp method. ${ }^{22}$ Insulin (Humulin R, Eli Lilly, Indianapolis, IN, USA), bound to albumin, was administered intravenously at a rate of $40 \mathrm{mU} / \mathrm{m}^{2} / \mathrm{min}$ for $120 \mathrm{~min}$. Blood was drawn by intravenous catheter in a heated vein, and glucose concentrations were measured at 5-min intervals. Infusion of $20 \%$ dextrose was adjusted to maintain a whole-blood glucose level of $90 \mathrm{mg} / \mathrm{dL}$. Peripheral glucose uptake ( $M$ value) was calculated according to the method of DeFronzo et al. ${ }^{22}$, based on steady-state glucose infusion rates. 
Body Composition Total body fat and lean body mass were measured by dual-energy X-ray absorptiometry (Hologics Discovery Wi, Bedford, MA, USA). Scanning was not performed in subjects who weighed $>350 \mathrm{lbs}$, the weight limit for the scanner.

Surgery The participants assigned to immediate surgery were discharged from the CRC and admitted for surgery the next day. The RYGB was performed in a standardized fashion by one author (GC); the technique has been described in detail previously. ${ }^{23,24}$ In brief, RYGB was performed laparoscopically with six to seven ports. A 3.5$\mathrm{mm}$ linear stapler transected the stomach to create a $30-\mathrm{mL}$ gastric pouch. An antecolic gastrojejunostomy route was always used. A circular gastrojejunal anastomosis with a 25-mm stapler was used. A biliopancreatic limb of $50 \mathrm{~cm}$ and an alimentary limb of $100 \mathrm{~cm}$ were measured, and a completely stapled side-to-side jejunojejunostomy was created. Patients were discharged on postoperative day 2 , and none had perioperative complications.

Participants were then followed as outpatients for 14 days, during which they consumed a standardized lowcalorie diet: Optifast HP (Novartis Nutrition Corporation), which provides $800 \mathrm{kcal} /$ day $(25 \%$ carbohydrate, $48 \%$ protein, and $27 \%$ fat). Different flavors were available, and participants were allowed to consume no-calorie, noncarbonated soft drinks and water ad libitum. They were given prepackaged servings and instructed to follow a specific feeding schedule. Each participant had met with the CRC dietitian during the baseline inpatient admission for individualized instructions regarding the diet and counseling. During the 14-day outpatient period, participants were asked to fill out daily logs of all food, water, and drinks ingested and were contacted every other day by a research fellow or coordinator from the Bariatric Surgery Clinic. Adherence to the diet was assessed by alternate-day phone calls from the research dietitian.

Follow-up in Patients Undergoing Diet Alone After completing the baseline evaluation and discharge from the CRC, participants assigned to the diet group started the 14-day diet period at home, following the identical diet routine as described for the RYGB group above.

Follow-up Metabolic Assessments (Visit 2 and Visit 3) After 14 days, all participants were readmitted to the CRC and underwent the same metabolic assessments performed at baseline (visit 2, V2). They were then discharged and continued their standard medical treatment. Six participants in the diet group underwent RYGB after completing the V2 assessment. A total of 12 subjects (nine originally assigned to RYGB and three to diet who subsequently underwent RYGB) had a third inpatient evaluation 6 months after RYGB (visit 3, V3).
Laboratory Analyses Whole-blood and plasma glucose levels were measured by the glucose oxidase method (YSI 2300 STAT-Plus Glucose Analyzer, YSI Inc., Yellow Springs, OH, USA). Serum insulin concentrations were measured by radioimmunoassay (Millipore, St. Charles, MO, USA). Active GLP-1 and GIP concentrations were measured by enzyme-linked immunosorbent assay (Millipore, St. Charles, MO, USA). The homeostasis model assessment of insulin resistance (HOMA-IR) was calculated as follows: fasting plasma glucose (millimoles per liter) $\times$ fasting serum insulin (microunits per milliliter) $/ 22.5 .^{25}$

\section{Statistical Analysis}

Data are summarized as mean and standard deviation unless otherwise stated. The unadjusted association of proportions and the distribution of continuous variables between groups and the association of each variable with outcomes were determined by two-sided $t$ test and chi-square tests. Area under the curve (AUC) was calculated using the trapezoidal rule. Linear associations were measured using the Pearson's correlation coefficient. Statistical significance was considered to be $p<0.05$. SPSS, version 13.0.1 (SPSS Inc, Chicago, IL, USA), was used for all statistical analyses.

\section{Results}

From October 2007 to January 2009, 59 patients met the study criteria, 28 initially agreed to participate, and 22 completed the evaluation procedures. Twelve patients were randomized or assigned to RYGB followed by standardized caloric restriction (RYGB) and ten to caloric restriction only (diet). The two groups did not differ with respect to baseline demographics, body composition (Table 1), fasting glucose and insulin, HOMA-IR, peripheral glucose disposal ( $M$ value), or hormonal secretion during the MTT (Table 2).

Table 1 Demographic and Clinical Characteristics of the RYGB and Diet Groups

\begin{tabular}{llll}
\hline & $\begin{array}{l}\text { RYGB } \\
(n=12)\end{array}$ & $\begin{array}{l}\text { Diet only } \\
(n=10)\end{array}$ & $p$ value \\
\hline Female/Male & $9: 3$ & $6: 4$ & 0.65 \\
Age (years) & $47.4 \pm 8.7$ & $40.2 \pm 13.4$ & 0.16 \\
Weight $(\mathrm{kg})$ & $138.0 \pm 21.6$ & $134.7 \pm 16.9$ & 0.70 \\
BMI $\left(\mathrm{kg} / \mathrm{m}^{2}\right)$ & $48.4 \pm 6.8$ & $48.3 \pm 6.6$ & 0.99 \\
\% Excess body weight & $55.4 \pm 6.4$ & $55.3 \pm 6.8$ & 0.96 \\
\% Fat (by DEXA) & $48.6 \pm 6.8$ & $46.8 \pm 4.7$ & 0.53
\end{tabular}

$R Y G B$ Roux-en-Y gastric bypass surgery, $B M I$ body mass index, $D E X A$ dual-energy $\mathrm{X}$-ray absorptiometry 
Table 2 Changes in Body Composition, and Baseline and Changes in Fasting Glucose and Insulin, HOMA-IR, AUCs for Insulin, GLP-1, and GIP During a Meal Tolerance Test at Baseline and 14 days

\begin{tabular}{lllr}
\hline & $\begin{array}{l}\text { RYGB } \\
(N=12)\end{array}$ & $\begin{array}{l}\text { Diet only } \\
(N=10)\end{array}$ & $\begin{array}{l}p \\
\text { value }\end{array}$ \\
\hline Weight loss (kg) & $9.9 \pm 2.4$ & $8.2 \pm 2.3$ & 0.11 \\
\% Excess weight loss & $12.7 \pm 2.4$ & $10.9 \pm 2.8$ & 0.12 \\
\% of weight lost as fat & $40.4 \pm 16.2$ & $29.9 \pm 16.8$ & 0.22 \\
Fasting glucose (mg/dL), baseline & $94.8 \pm 12.0$ & $99.6 \pm 14.7$ & 0.41 \\
Change in fasting glucose & $-7.8 \pm 10.1$ & $-13.1 \pm 17.7$ & 0.40 \\
$p$ value & 0.02 & 0.04 & \\
Fasting insulin ( $\mu$ U/mL), baseline & $22.4 \pm 14.4$ & $34.1 \pm 20.1$ & 0.15 \\
Change in fasting insulin & $-7.7 \pm 7.5$ & $-13.7 \pm 15.9$ & 0.29 \\
$p$ value & $<0.01$ & 0.02 & \\
HOMA-IR, baseline & $5.1 \pm 2.9$ & $8.9 \pm 7.0$ & 0.14 \\
Change in HOMA-IR & $-1.9 \pm 1.4$ & $-4.6 \pm 6.2$ & 0.22 \\
$p$ value & 0.01 & 0.04 & \\
AUC insulin, baseline & $196 \pm 70.6$ & $276 \pm 89.3$ & 0.03 \\
Change in AUC insulin & $33 \pm 131.6$ & $-21 \pm 114.5$ & 0.32 \\
$p$ value & 0.40 & 0.57 & \\
AUC GLP-1, baseline & $5.1 \pm 4.1$ & $3.5 \pm 1.2$ & 0.59 \\
Change in GLP-1 AUC & $13.4 \pm 11.0$ & $0.5 \pm 1.6$ & $<0.01$ \\
$p$ value & $<0.01$ & 0.24 & \\
AUC GIP, baseline & $226.0 \pm 93.1$ & $201.0 \pm 67.4$ & 0.59 \\
Change in GIP AUC & $-11.4 \pm 80.1$ & $132.0 \pm 40.3$ & $<0.01$ \\
$p$ value & 0.63 & 0.02 & \\
\hline
\end{tabular}

HOMA-IR homeostasis model assessment, $A U C$ area under the curve, MTT meal tolerance test

At baseline, peripheral glucose uptake determined by the euglycemic-hyperinsulinemic clamp was profoundly impaired in all subjects; average $M$ value was about one third of that for lean controls in our laboratory $(2.1 \pm 0.9$ vs. $7.6 \pm$ $2.3 \mathrm{mg} / \mathrm{kg} / \mathrm{min}, p<0.01)^{26}$

\section{Metabolic Changes}

After the 14-day diet period, the magnitude of weight loss and changes in body composition did not differ between groups. Fasting glucose, insulin, and HOMA-IR decreased similarly in both groups (Table 2). $M$ values did not change in either group (RYGB $(n=10), \mathrm{V} 1=2.4 \pm 0.9$ vs. V2 $=2.3 \pm$ $0.7 \mathrm{mg} / \mathrm{kg} / \mathrm{min}, p=0.80$ and diet $(n=8), \mathrm{V} 1=1.8 \pm 1.0 \mathrm{vs}$. $\mathrm{V} 2=2.0 \pm 1.0 \mathrm{mg} / \mathrm{kg} / \mathrm{min}, p=0.57$; Fig. 1).

Insulin secretion over the 3 -h postmeal period was similar for V1 vs. V2 in both groups at baseline (Table 2), but after RYGB, serum insulin levels in the first $30 \mathrm{~min}$ after the meal were higher than for the diet group (RYGB, $\mathrm{V} 1=107 \pm 44$ vs. $\mathrm{V} 2=181 \pm 137 \mu \mathrm{U} / \mathrm{mL} ; p=0.01$ and diet, $\mathrm{V} 1=117 \pm 40$ vs. $\mathrm{V} 2=133 \pm 90 \mu \mathrm{U} / \mathrm{mL}, p=0.83$; Fig. 2). GLP-1 postmeal AUC increased significantly following
RYGB, whereas it did not change after diet only (Table 2). GIP AUC increased after diet, whereas the values remained low in the RYGB patients (Table 2). The increase in GLP-1 AUC in the RYGB group paralleled the early increase in insulin release after RYGB (Fig. 2).

\section{Metabolic Changes 6 Months After RYGB}

Six participants in the diet group underwent RYGB after completing V2 assessment. A total of 12 subjects underwent a third inpatient evaluation 6 months after RYGB (V3): nine subjects from the original RYGB group and three from the diet group who underwent RYGB after completing V1 and V2. Six months after RYGB, this group of subjects had sustained significant weight loss (weight loss $=28.4 \pm 4.6 \mathrm{~kg} ; \mathrm{EWL}=49.7 \%, p<0.01 \mathrm{vs}$. V1) of which $74.5 \%$ was fat and had significant changes in fasting glucose, insulin, and HOMA-IR compared to baseline (Table 3). The magnitude of changes in fasting glucose, insulin, and HOMA-IR observed at 6 months (V1-3) for the patients originally assigned to RYGB was similar to changes observed at 14 days (V1-2): change in glucose $\mathrm{V} 1-2=-7.8 \pm 2.9$ vs. $\mathrm{V} 1-3=-9.6 \pm 4.1 \mathrm{mg} / \mathrm{dL}, p=0.73$; change in insulin $\mathrm{V} 1-2=-7.7 \pm 2.2$ vs. $\mathrm{V} 1-3=-12.6 \pm$ $3.5 \mu \mathrm{IU} / \mathrm{mL}, p=0.25$; and change in HOMA-IR V1-2= $-1.9 \pm 0.4$ vs. $\mathrm{V} 1-3=-3.0 \pm 0.7, p=0.25$. The changes in the postmeal serum levels and AUCs of GLP-1, GIP, and insulin 6 months after RYGB remained similar to those observed at 14 days (Tables 2 and 3). In contrast, peripheral glucose uptake ( $M$ value) increased in all subjects at 6 months, reaching the upper boundary for the lowermost quartile of values in healthy control subjects (Fig. 3). Notably, the changes in $M$ values correlated significantly with the magnitude of weight lost $(r=0.68, p=0.02$; Fig. 4).

\section{Discussion}

Operations such as RYGB that bypass the duodenum and/or stomach, thus allowing for early delivery of the food bolus to the small intestine and preventing food bolus contact with the duodenum, offer a unique opportunity for identifying weight loss independent mechanisms for resolution of diabetes. ${ }^{9}$ Consequently, a variety of novel surgical and endoscopic gastrointestinal procedures are under investigation for use as therapeutic options in treating T2DM. ${ }^{27-31}$ Critical unanswered questions remain as to whether or to what degree the altered patterns of gut and pancreatic hormone secretion known to occur with bypass operations bolster beta cell function and ultimately improve peripheral glucose disposal and promote resolution of T2DM independent of weight loss. ${ }^{9}$ The fact remains, however, that bariatric operations that result in greater 

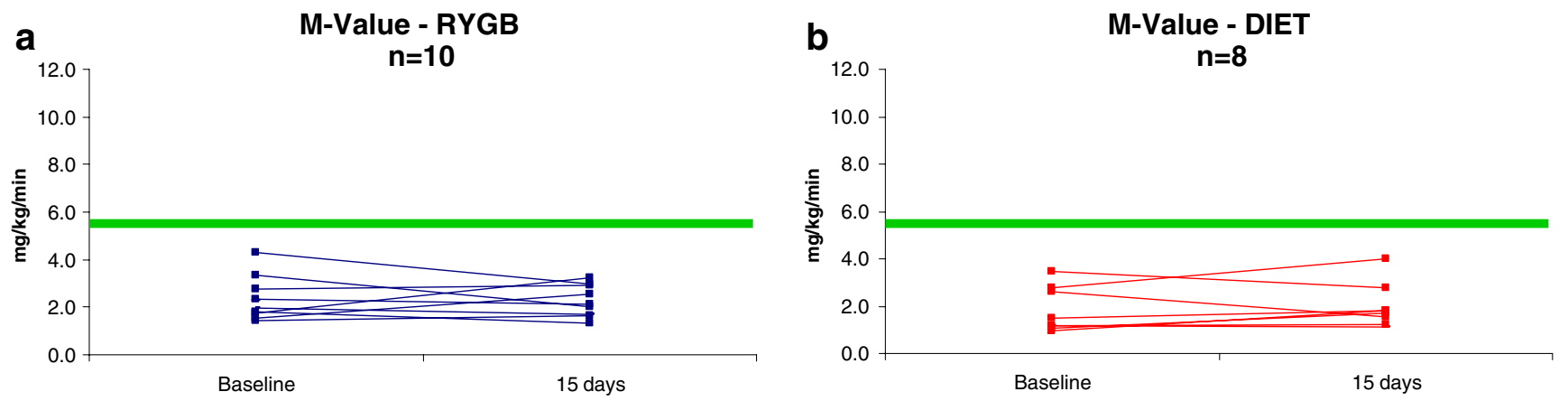

Figure 1 Peripheral glucose uptake ( $M$ value) by euglycemic-hyperinsulinemic clamp before (baseline) and 14 days after either RYGB (a) or caloric restriction (b). The horizontal line indicates the upper boundary for the lower-most quartile of values seen in healthy control subjects. ${ }^{26}$

weight loss are still associated with greater rates of T2DM improvement or cure. ${ }^{1}$ In a recent systematic review and meta-analysis, Buchwald and colleagues ${ }^{1}$ showed that excess weight loss and diabetes resolution in the first 12 months after surgery were the highest for patients undergoing biliopancreatic diversion/duodenal switch (BPD/DS; 64\% excess weight loss, 95\% diabetes resolution) followed by RYGB (60\% excess weight loss, $80 \%$ diabetes resolution) and the least for banding procedures (46\% excess weight loss, 57\% diabetes resolution). However, BPD/DS and RYGB may impact and alter mechanisms other than weight loss that play an independent role in improving insulin resistance and allow for this high rate of resolution of T2DM. The intricate interplay among insulin sensitivity/resistance, glucose metabolism, and insulin secretion is affected by many factors: beta cell function; quality, distribution, and total fat mass; energy balance and the magnitude of calorie restriction; hepatic glucose and insulin metabolism and kinetics; quality and quantity of nutrient intake and absorption; associated diseases and stressors such as sleep apnea, liver fat, and adipocytokines; and gut and pancreatic hormone secretion and metabolism, among others. ${ }^{9,32}$

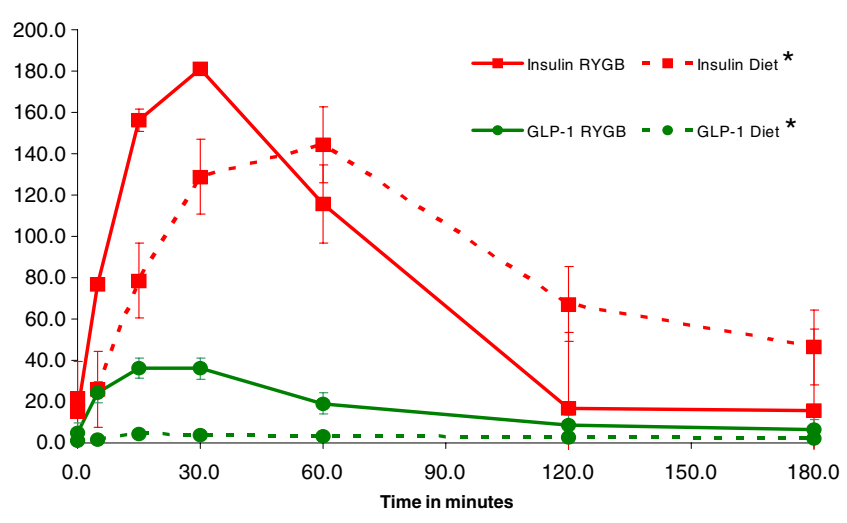

RYGB= Roux-en-Y gastric bypass surgery

* Insulin in microU/mL ** GLP-1 in pM

Figure 2 Insulin and GLP-1 secretion after a meal, 14 days after RYGB and calorie restriction or diet alone.
In this study, we sought to delineate short-term changes in peripheral glucose disposal, fasting measures of glucose and insulin, gut and pancreatic hormone secretion in response to a meal challenge, and body composition, while controlling for energy balance, and to evaluate these same changes after more substantial weight loss had occurred 6 months after RYGB. We confirmed previous studies in showing that, after RYGB, the pattern of gut and pancreatic hormone secretion following a meal is altered compared to

Table 3 Baseline Fasting Glucose, Insulin, HOMA-IR, AUCs for Insulin, GLP-1, and GIP During a Meal Tolerance Test and Changes 6 Months After RYGB

\begin{tabular}{ll}
\hline & RYGB $\left(N=12^{\mathrm{a}}\right)$ \\
\hline Fasting glucose $(\mathrm{mg} / \mathrm{dL})$, baseline & $91.9 \pm 10.3$ \\
Change in fasting glucose & $-9.6 \pm 14.2$ \\
$p$ value & 0.03 \\
Fasting insulin $(\mu \mathrm{U} / \mathrm{mL})$, baseline & $22.9 \pm 14.0$ \\
Change in fasting insulin & $-12.6 \pm 12.3$ \\
$p$ value & $<0.01$ \\
HOMA-IR, baseline & $5.1 \pm 2.8$ \\
Change in HOMA-IR & $-2.9 \pm 2.6$ \\
$p$ value & $<0.01$ \\
AUC insulin, baseline & $191 \pm 62$ \\
Change in AUC Insulin & $2.1 \pm 103$ \\
$p$ value & 0.95 \\
AUC GLP-1, baseline & $3.7 \pm 2.2$ \\
Change in GLP-1 AUC & $11.6 \pm 5.4$ \\
$p$ value & $<0.01$ \\
AUC GIP, baseline & $207.4 \pm 65.8$ \\
Change in GIP AUC & $-4.5 \pm 89.9$ \\
$p$ value & 0.87 \\
\hline
\end{tabular}

HOMA-IR homeostasis model assessment, $A U C$ area under the curve, MTT meal tolerance test

${ }^{\text {a }}$ A total of 12 subjects underwent a third inpatient evaluation 6 months after RYGB (visit 3): nine subjects from the original RYGB group and three from the diet group who underwent RYGB after completing V1 and V2 


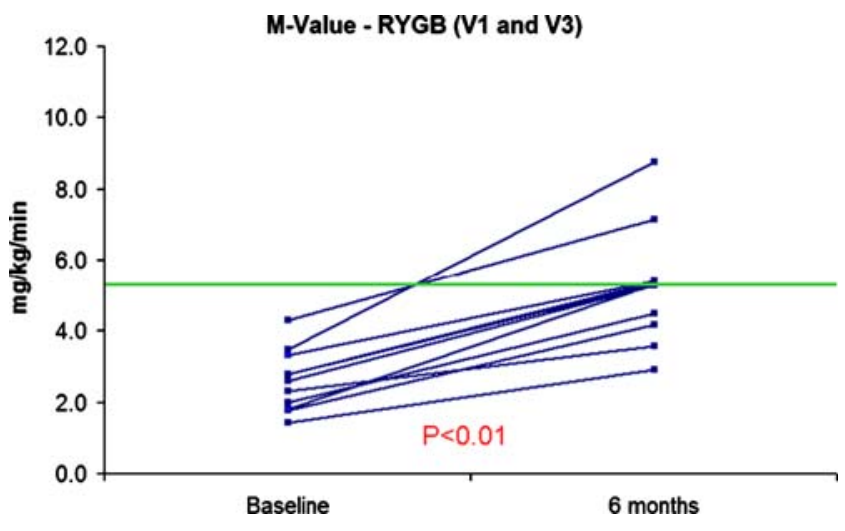

Figure 3 Peripheral glucose uptake ( $M$ value) by euglycemichyperinsulinemic clamp before and 6 months after RYGB $(n=11)$.

controls $^{10,11,30,33}$ and that RYGB patients have early improvements in fasting glucose, fasting insulin, and, thus, in calculated HOMA-IR. Notably, the magnitude of improvement in fasting glucose homeostasis was similar in the group that underwent diet alone, indicating that weight loss, rather than the surgical procedure, results in these early changes. We also found that soon after RYGB and before massive weight loss occurs, peripheral glucose disposal (measured by euglycemic-hyperinsulinemic clamp) was not improved. Taken together, these results suggest that in the nondiabetic morbidly obese with severe insulin resistance, the short-term effects of gastric bypass surgery can improve glucose homeostasis under fasting conditions before substantial weight loss occurs but are not sufficient to improve peripheral glucose disposal during hyperinsulinemia. Other investigators, using a variety of techniques to study glucose metabolism, have documented

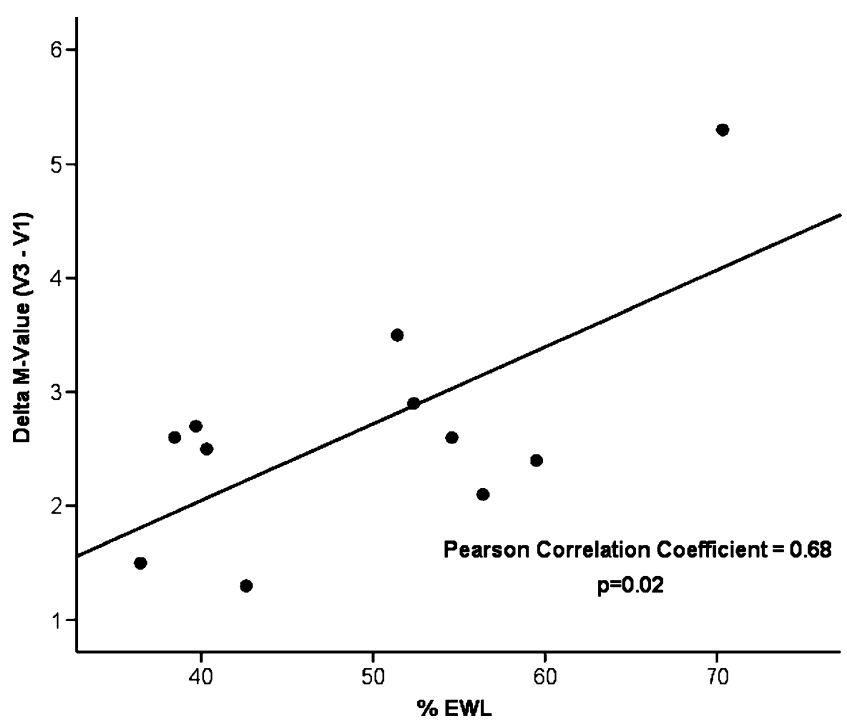

Figure 4 Correlation in between changes in peripheral glucose uptake ( $M$ values) and percent excess weight loss at 6 months after RYGB $(n=11)$. improvements in insulin resistance from 1 week to 1 month after $\mathrm{BPD} / \mathrm{DS}^{34}$ and also RYGB with weight loss varying from 9 to $15 \mathrm{~kg} .{ }^{34-37}$ In contrast to our study, these studies were done in diabetic subjects and most were performed without a diet-only control group. Nevertheless, these differences support the notion that diabetic patients may respond differently to these procedures than nondiabetics.

The altered pattern of gut hormone secretion may result in amelioration of glucose disposal independently of weight loss by many mechanisms. For example, GLP-1 reduces elevated fasting and postprandial blood glucose levels in diabetic humans; leads to glucose-dependent insulin secretion, induction of beta-cell proliferation and expansion of the beta-cell mass, and enhanced resistance to beta-cell apoptosis; inhibits gastric emptying and acid secretion; and reduces food ingestion and glucagon secretion, among other functions. $^{20,38} \mathrm{We}$ and others demonstrated that RYGB is associated with an increased release of postprandial GLP-1. ${ }^{13,20,33,39}$ While the more rapid delivery of glucose and other nutrients to the proximal intestine may partially explain the rapid and robust insulin and GLP-1 responses following RYGB, the magnitude of the exaggeration of the GLP-1 response suggests that other unique features with RYGB also contribute to this increase. In another example, GIP, which is released from the duodenum and proximal small bowel $\mathrm{K}$-cells in response to glucose and fat ingestion, augments glucose-stimulated insulin secretion in healthy humans but almost completely loses its insulinotropic effect in patients with T2DM. ${ }^{20,33}$ Others have shown a blunted recovery of GIP levels in obese diabetic patients, but not in obese nondiabetics, after RYGB. ${ }^{17,33,40}$ Patients in our current study, although not classified as diabetics, had markedly impaired glucose disposal, and the recovery of GIP secretion in diet-only subjects after minimal weight loss and maintenance of low levels of GIP in the RYGB group were similar to those observed in diabetics in other studies. ${ }^{17,33,40}$

A unique strength of our study was the use of the hyperinsulinemic-euglycemic clamp and repeated testing under controlled conditions. The clamp technique is widely accepted as the reference standard for directly determining insulin sensitivity and peripheral glucose disposal in humans $^{41}$ and offers, in the research setting, significant advantages over the commonly used technique for assessing insulin sensitivity, such as HOMA-IR. The hyperinsulinemic-euglycemic clamp leads to a steady-state condition in which the glucose infusion rate during the clamp must be equal to the glucose disposal rate. Thus, the clamp estimates insulin sensitivity/resistance in humans and directly measures peripheral glucose disposal at a given level of insulinemia under steady-state conditions. ${ }^{22,41}$ In addition, the glucose clamp has excellent test characteristics. Peripheral glucose uptake typically has a coefficient 
of variation of 0.10 and a discriminant ratio of 6 (a measurement of both reproducibility and the ability to distinguish individual results). ${ }^{42}$ On the other hand, HOMA-IR, which has been used in many other studies of the metabolic effects of bariatric surgery, is a simple surrogate index for insulin resistance that is derived from blood insulin and glucose concentrations under fasting conditions and reflects mostly hepatic insulin sensitivity. The main limitations of the glucose clamp approach are that it is time-consuming, labor-intensive, expensive, and requires an experienced operator to manage the technical difficulties, but these were overcome by the use of CRC resources and the experienced group of endocrinologists. Moreover, because glucose and insulin are administered parenterally during the clamp, thus bypassing the gut and splanchnic metabolism, this technique measured peripheral glucose uptake independent of any potential influence of altered nutrient delivery to the small intestine or changes in incretin secretion following RYGB.

Evidence from our study and others leaves little doubt that calorie restriction alone can improve fasting glucose and insulin levels and is an important factor leading to the rapid changes observed after RYGB. However, as detailed above, RYGB provides mechanisms that are independent of weight loss and impact and/or bolster the ability of morbidly obese patients to experience a more rapid amelioration of glucose metabolism. However, fasting glucose and insulin measurements, which are usually obtained as a surrogate for insulin sensitivity in most studies performed to date and were the measures that improved similarly at 14 days after RYGB or calorie restriction only, are mostly a reflection of hepatic insulin sensitivity. The $M$ value obtained during the clamp study provides the best estimate of peripheral glucose homeostasis, and as we demonstrate, $M$ values improved only after significant weight loss and correlated with the magnitude of weight loss.

Limitations of our study include small differences between RYGB and diet groups in gender distribution, weight loss, percentage of weight loss as fat, average daily energy intake, and possible differences in diet absorption that, although not statistically significant, when combined may have impacted the results. Nevertheless, these differences in changes would have led to improvement in glucose metabolism in the RYGB group. On the other hand, the surgical insult and trauma of a laparoscopic operation for RYGB may have had a negative impact, in the 14 day analyses, on glucose homeostasis and disposal. Lastly, we may have not been able to identify a differential and earlier improvement in peripheral glucose disposal in the RYGB group because we studied only nondiabetic participants and studied them only at three time points. Although our participants were profoundly insulin resistant, they may have had effective counter-regulatory mechanisms that successfully forestalled progression to diabetes, and thus, we may have observed a different result had we studied patients with diabetes.

With these limitations in view, we conclude that in nondiabetic morbidly obese subjects under similar caloric restriction and weight loss, peripheral glucose disposal is not improved early after RYGB or calorie restriction. Improvement in peripheral glucose disposal following RYGB was observed only after substantial weight loss had occurred and correlated with the magnitude of weight lost. These findings suggest that weight loss is a critical component for complete restoration of glucose homeostasis in the morbidly obese with insulin resistance.

Acknowledgments This publication was supported by Grant Number KL2 RR024130 from the National Center for Research Resources (NCRR), a component of the NIH and NIH Roadmap for Medical Research (GMC), and by NIH/NCRR UCSF-CTSI Grant Number UL1 RR024131. We are also grateful for the assistance of the SFGH-CRC nursing, dietary, and laboratory staff, especially Laurie Herraiz, RD, Veronica Monti, RD, and Viva Tai, MPH, RD; James Graham, PhD, of the University of California Davis, who coordinated the hormone analyses; and Eric Vittinghoff, $\mathrm{PhD}$, for assistance with the statistical analyses.

Open Access This article is distributed under the terms of the Creative Commons Attribution Noncommercial License which permits any noncommercial use, distribution, and reproduction in any medium, provided the original author(s) and source are credited.

\section{References}

1. Buchwald H, Estok R, Fahrbach $\mathrm{K}$ et al. Weight and type 2 diabetes after bariatric surgery: systematic review and metaanalysis. Am J Med 2009;122(3):248-256. e245.

2. Pories WJ, Swanson MS, MacDonald KG et al. Who would have thought it? An operation proves to be the most effective therapy for adult-onset diabetes mellitus. Ann Surg 1995;222(3):339-350. discussion 350-332.

3. Buchwald H. Consensus conference statement bariatric surgery for morbid obesity: health implications for patients, health professionals, and third-party payers. Surg Obes Relat Dis 2005; 1(3):371-381.

4. Buchwald H, Avidor Y, Braunwald E et al. Bariatric surgery: a systematic review and meta-analysis. JAMA 2004;292(14):1724 1737.

5. Nguyen NT, Hinojosa M, Fayad C, Varela E, Wilson SE. Use and outcomes of laparoscopic versus open gastric bypass at academic medical centers. J Am Coll Surg 2007;205(2):248-255.

6. Bikman BT, Zheng D, Pories WJ et al. Mechanism for improved insulin sensitivity after gastric bypass surgery. J Clin Endocrinol Metab 2008;93(12):4656-4663.

7. Borg CM, le Roux CW, Ghatei MA, Bloom SR, Patel AG, Aylwin SJ. Progressive rise in gut hormone levels after Roux-en-Y gastric bypass suggests gut adaptation and explains altered satiety. Br J Surg 2006;93(2):210-215.

8. Cummings DE, Flum DR. Gastrointestinal surgery as a treatment for diabetes. JAMA 2008;299(3):341-343.

9. Ferrannini E, Mingrone G. Impact of different bariatric surgical procedures on insulin action and beta-cell function in type 2 diabetes. Diabetes Care 2009;32(3):514-520. 
10. Korner J, Inabnet W, Febres G et al. Prospective study of gut hormone and metabolic changes after adjustable gastric banding and Roux-en-Y gastric bypass. Int J Obes (Lond) 2009;33(7):786-795.

11. le Roux CW, Welbourn R, Werling $M$ et al. Gut hormones as mediators of appetite and weight loss after Roux-en-Y gastric bypass. Ann Surg 2007;246(5):780-785.

12. Rubino F. Is type 2 diabetes an operable intestinal disease? A provocative yet reasonable hypothesis. Diabetes Care. 2008;31( Suppl 2):S290-296.

13. Laferrere B, Heshka S, Wang $\mathrm{K}$ et al. Incretin levels and effect are markedly enhanced 1 month after Roux-en-Y gastric bypass surgery in obese patients with type 2 diabetes. Diabetes Care 2007;30(7):1709-1716.

14. Laferrere B, Teixeira J, McGinty $\mathrm{J}$ et al. Effect of weight loss by gastric bypass surgery versus hypocaloric diet on glucose and incretin levels in patients with type 2 diabetes. J Clin Endocrinol Metab 2008;93(7):2479-2485.

15. Cummings DE, Overduin J, Foster-Schubert KE. Gastric bypass for obesity: mechanisms of weight loss and diabetes resolution. J Clin Endocrinol Metab 2004;89(6):2608-2615.

16. Korner J, Inabnet W, Conwell IM et al. Differential effects of gastric bypass and banding on circulating gut hormone and leptin levels. Obesity (Silver Spring) 2006;14(9):1553-1561.

17. Rubino F, Gagner M, Marescaux J. Surgical treatment of type 2 diabetes mellitus. Lancet 2001;358(9282):668-669.

18. Rodieux F, Giusti V, D'Alessio DA, Suter M, Tappy L. Effects of gastric bypass and gastric banding on glucose kinetics and gut hormone release. Obesity (Silver Spring) 2008;16(2):298-305.

19. Cummings DE, Overduin J, Shannon MH, Foster-Schubert KE. Hormonal mechanisms of weight loss and diabetes resolution after bariatric surgery. Surg Obes Relat Dis 2005;1(3):358-368.

20. Baggio LL, Drucker DJ. Biology of incretins: GLP-1 and GIP. Gastroenterology 2007;132(6):2131-2157.

21. Batterham RL, Heffron H, Kapoor S et al. Critical role for peptide YY in protein-mediated satiation and body-weight regulation. Cell Metab 2006;4(3):223-233.

22. DeFronzo RA, Tobin JD, Andres R. Glucose clamp technique: a method for quantifying insulin secretion and resistance. Am J Physiol 1979;237(3):E214-223.

23. Campos GM, Ciovica R, Rogers SJ et al. Spectrum and risk factors of complications after gastric bypass. Arch Surg 2007;142 (10):969-975. discussion 976.

24. Campos GM, Rabl C, Mulligan K et al. Factors associated with weight loss after gastric bypass. Arch Surg 2008;143(9):877-883. discussion 884 .

25. Matthews DR, Hosker JP, Rudenski AS, Naylor BA, Treacher DF, Turner RC. Homeostasis model assessment: insulin resistance and beta-cell function from fasting plasma glucose and insulin concentrations in man. Diabetologia 1985;28(7):412-419.

26. Rao MN, Mulligan K, Schambelan M. HIV infection and diabetes. In Poretsky L, ed. Principles of Diabetes Mellitus. Norwell: Springer, 2009.

27. Ashrafian H, le Roux CW. Metabolic surgery and gut hormones-a review of bariatric entero-humoral modulation. Physiol Behav 2009;97:620-631.

28. Ellsmere JC, Thompson CC, Brugge WR et al. Endoscopic interventions for weight loss surgery. Obesity (Silver Spring) 2009;17(5):929-933.

29. Fetner R, McGinty J, Russell C, Pi-Sunyer FX, Laferrere B. Incretins, diabetes, and bariatric surgery: a review. Surg Obes Relat Dis. 2005;1(6):589-597. discussion 597-588.

30. Rubino F, Forgione A, Cummings DE et al. The mechanism of diabetes control after gastrointestinal bypass surgery reveals a role of the proximal small intestine in the pathophysiology of type 2 diabetes. Ann Surg 2006;244(5):741-749.
31. Smith BR, Schauer P, Nguyen NT. Surgical approaches to the treatment of obesity: bariatric surgery. Endocrinol Metab Clin North Am 2008;37(4):943-964.

32. Cummings DE. Endocrine mechanisms mediating remission of diabetes after gastric bypass surgery. Int J Obes (Lond) 2009;33 (Suppl 1):S33-40.

33. Korner J, Bessler M, Inabnet W, Taveras C, Holst JJ. Exaggerated glucagon-like peptide-1 and blunted glucose-dependent insulinotropic peptide secretion are associated with Roux-en-Y gastric bypass but not adjustable gastric banding. Surg Obes Relat Dis 2007;3(6):597-601.

34. Guidone C, Manco M, Valera-Mora E et al. Mechanisms of recovery from type 2 diabetes after malabsorptive bariatric surgery. Diabetes 2006;55(7):2025-2031.

35. Morinigo R, Casamitjana R, Delgado $S$ et al. Insulin resistance, inflammation, and the metabolic syndrome following Roux-en-Y gastric bypass surgery in severely obese subjects. Diabetes Care 2007;30(7):1906-1908.

36. Perugini RA, Quarfordt SH, Baker S, Czerniach DR, Litwin DE, Kelly JJ. Metabolic characterization of nondiabetic severely obese patients undergoing Roux-en-Y gastric bypass: preoperative classification predicts the effects of gastric bypass on insulin-glucose homeostasis. J Gastrointest Surg 2007;11(9): 1083-1090.

37. Salinari S, Bertuzzi A, Iaconelli A, Manco M, Mingrone G. Twenty-four hour insulin secretion and beta cell NEFA oxidation in type 2 diabetic, morbidly obese patients before and after bariatric surgery. Diabetologia 2008;51(7):1276-1284.

38. Hagemann D, Holst JJ, Gethmann A, Banasch M, Schmidt WE, Meier JJ. Glucagon-like peptide 1 (GLP-1) suppresses ghrelin levels in humans via increased insulin secretion. Regul Pept 2007;143(1-3):64-68.

39. le Roux CW, Aylwin SJ, Batterham RL et al. Gut hormone profiles following bariatric surgery favor an anorectic state, facilitate weight loss, and improve metabolic parameters. Ann Surg 2006;243(1):108 114.

40. Rubino F, Gagner M. Potential of surgery for curing type 2 diabetes mellitus. Ann Surg 2002;236(5):554-559.

41. Muniyappa R, Lee S, Chen H, Quon MJ. Current approaches for assessing insulin sensitivity and resistance in vivo: advantages, limitations, and appropriate usage. Am J Physiol Endocrinol Metab 2008;294(1):E15-26.

42. Mather KJ, Hunt AE, Steinberg HO et al. Repeatability characteristics of simple indices of insulin resistance: implications for research applications. J Clin Endocrinol Metab 2001;86(11): $5457-5464$

\section{Discussant}

Dr. Bruce M. Wolfe (Portland, OR): Dr. Campos and colleagues demonstrated that 2 weeks postgastric bypass, GLP-1 and insulin secretion were enhanced, but peripheral insulin resistance as reflected by glucose uptake did not decrease until substantial weight loss occurred at 6 months postoperatively. I have the following questions: First, you indicated that all subjects demonstrated insulin resistance prior to operations. Would you comment on the importance of this finding?

Second, HOMA, a measure of insulin resistance calculated from fasting glucose and insulin decreased at 2 weeks, but the clamp derived $M$ score of glucose uptake and 
peripheral insulin resistance did not. Is HOMA a useful parameter in these studies or will further studies require the extensive and burdensome clamp studies?

Finally, are you confident that the insulin infusion was effective in shutting down hepatic gluconeogenesis allowing you to draw conclusions regarding peripheral glucose uptake?

Closing Discussant

Dr. Guilherme M. Campos (San Francisco, CA): Thank you, Dr. Wolfe. Your questions highlight the need to clearly understand the definition of diabetes and prediabetes and that diabetes is a spectrum of a disease. What is interesting in our patient population of morbidly obese individuals is that none had a diagnosis of diabetes. All their fasting glucose measures were below 105 and with normal hemoglobin A1c, thus fitting the current criteria for nondiabetics. However, when we studied and challenged them using the hyperinsulinemic-euglycemic clamp, they uniformly had peripheral insulin resistance and poor peripheral glucose disposal. How to incorporate and apply these tests in other studies? The clamp technique is widely accepted as the reference standard for directly determining insulin sensitivity and peripheral glucose disposal or uptake in humans and offers, in the research setting, significant advantages over the commonly used technique for assessing insulin sensitivity, such as fasting measurements like
HOMA-IR. However, it cannot be used in large epidemiological studies as it is indeed labor-intensive and expensive. Thus, surrogate indices of insulin resistance, such as HOMA-IR, are an acceptable alternative, but as shown in our study, it will not identify all patients with impaired glucose metabolism.

Lastly, was the amount of insulin chosen during the clamp effective in suppressing hepatic gluconeogenesis?-the answer is we do not know. We would have to have radiolabeled glucose tracer studies to study hepatic glucose production to see if the effect was indeed enough to suppress hepatic gluconeogenesis. But that does not affect our results and conclusions as we use the same amount of intravenous insulin in all three evaluations using standard dosing per square meter. So, even if hepatic gluconeogenesis was not suppressed, the observed values and changes are still valid and reliable.

Discussant

Dr. Nils Lambrecht (Los Angeles, CA): Do you have any data on gastric sleeve surgery because the physical removal of a large portion of the gastric oxyntic mucosa including most ghrelin containing endocrine cells may play a big role in changes in food intake and dietary behavior?

Closing Discussant

Dr. Guilherme M. Campos (San Francisco, CA): No, we do not have data on gastric sleeve. 